\title{
Doença Renal Aguda em gatos: conquistas e desafios
}

\author{
[Acute Kidney Disease in cats: achievements and challenges]
}

\section{"Revisão/Review"}

\author{
Francisco Antônio Félix Xavier Júnior ${ }^{1 *}$, Glayciane Bezerra Morais ${ }^{2}$, Marrie Silva Dutra ${ }^{2}$, \\ Mateus Mendes Freitas ${ }^{2}$, Steffi Lima Araujo ${ }^{1}$, Daniel de Araújo Viana ${ }^{3}$, \\ Janaina Serra Azul Monteiro Evangelista ${ }^{2}$
}

\author{
${ }^{1}$ Programa de Pós-Graduação em Ciências Veterinárias, Universidade Estadual do Ceará, Fortaleza-CE, Brasil. \\ ${ }^{2}$ Faculdade de Veterinária, Universidade Estadual do Ceará, Fortaleza-CE, Brasil. \\ ${ }^{3}$ Laboratório de Anatomia Patológica \& Patologia Clínica Veterinária, Fortaleza-CE, Brasil. \\ *Autor para correspondência/Corresponding author: E-mail: juniorfelix.medicoveterinario@gmail.com
}

\section{Resumo}

A doença renal aguda (DRA) refere-se a uma síndrome clínica associada a alta morbidade e mortalidade, fazendo parte da síndrome de disfunção de múltiplos órgãos. Em medicina veterinária, estudos sobre a incidência de casos com DRA ainda são pouco elucidados, e a fisiopatologia da lesão renal aguda (DRA) é complexa, sendo caracterizada pela rápida diminuição da função excretora renal e consequente acúmulo de produtos do metabolismo de nitrogênio. Nesse contexto, o objetivo desse estudo foi apresentar a DRA com dados atuais sobre etiologia, patogenia, diagnóstico e tratamento.

Palavras-chave: insuficiência renal aguda; lesão renal aguda; medicina felina.

\begin{abstract}
Acute kidney disease (AKI) is a clinical syndrome associated with high morbidity and mortality, and is part of multiple organ dysfunction syndrome. In veterinary medicine, studies have not fully elucidated the incidence of AKI cases, in particular because the pathophysiology of acute kidney injury is complex, being characterized by the rapid loss of kidney excretory function, causing accumulation of nitrogen metabolism products. In this context, the purpose of this study is to show the current concepts and new insights regarding AKI.
\end{abstract}

Keywords: acute renal failure; acute kidney injury; feline medicine.

\section{Introdução}

A doença renal aguda (DRA) representa um espectro de doença associado a um início súbito de lesão do parênquima renal, caracterizada, em geral, pela falha generalizada dos rins em atender às demandas excretórias, metabólicas e endócrinas do corpo, resultando em uremia aguda (UA) (Worwag e Langston, 2008).

A lesão renal aguda (DRA) se desenvolve a partir de múltiplas etiologias, incluindo isquemia prolongada, nefrotoxinas, doenças renais intrínsecas, causas infecciosas e obstrução ou ruptura do fluxo urinário (Worwag e Langston, 2008).
O modelo conceitual e atual da DRA identifica quatro componentes em sua evolução: fase de risco, rim apresenta-se normal, porém encontra-se um risco de lesão aumentado; fase intermediária de dano renal, presença de uma lesão funcional; insuficiência renal propriamente dita, diminuição da filtração glomerular e insuficiência renal e, por último, falência renal, o que pode levar à morte dependendo do dano inicial e da persistência dessa lesão (Bellomo et al., 2012). O objetivo do presente estudo foi discorrer uma revisão de literatura sobre a doença renal aguda em 
gatos e os conhecimentos acerca do assunto até o presente momento.

\section{Desenvolvimento \\ Doença Renal Aguda}

A DRA é uma síndrome clínica caracterizada por uma diminuição rápida (horas a dias) da função excretora renal, com acúmulo de produtos do metabolismo do nitrogênio, como por exemplo, a creatinina e a ureia, além de produtos de resíduos clinicamente não mensurados. Outras manifestações clínicas e laboratoriais comuns incluem oligúria, hipercalemia e hiperfosfatemia (Bonventre e Yang, 2011; Bellomo et al., 2012).

A lesão renal pode ser imperceptível, clinicamente, em estádios iniciais e culminar com a necessidade de terapia de substituição renal (hemodiálise, diálise peritoneal ou transplante renal) com o aparecimento de insuficiência evidente da função renal ou morte (Iris, 2016).

Existem 3 tipos de classificações quanto à localização da lesão de acordo com seus mecanismos básicos que levam à disfunção renal, sendo eles: DRA pré-renal, DRA renal e DRA pósrenal.

Na DRA pré-renal o rim está funcionalmente íntegro, mas a perfusão sanguínea encontra-se reduzida. Esta hipoperfusão renal é geralmente causada por hipovolemia aguda em casos de desidratação por perdas gastrointestinais ou hemorragia grave (Horkan et al., 2015). No entanto, há casos em que a volemia arterial efetiva está reduzida, como na insuficiência cardíaca congestiva grave e na cirrose. Esse quadro é reversível se o distúrbio volêmico for corrigido rapidamente. A manutenção prolongada dessa hipoperfusão renal pode causar lesão e necrose de células tubulares, principalmente nos segmentos renais localizados na medula externa (túbulo contorcido proximal e porção espessa da alça da Henle) (Kellum, 2008).

A DRA renal é caracterizada principalmente por necrose tubular aguda (NTA), embora possam aparecer outras alterações, às vezes sutis, como a condensação de cromatina nuclear, lesões de cristas mitocondriais e vacuolização citoplasmática, frequentemente visível apenas à microscopia eletrônica (Kellum, 2008).

$\mathrm{Na}$ DRA pós-renal o fluxo urinário é dificultado, ou mesmo interrompido, por obstrução mecânica das vias urinárias, devido, por exemplo, à presença de cálculos ou crescimento tumoral. Essa modalidade de DRA é potencialmente reversível caso seja realizada a desobstrução precoce das vias urinárias. No entanto, a recuperação pode ser mais difícil ou evoluir para necrose tubular aguda (NTA), se a duração do processo obstrutivo tiver sido demasiadamente longa (Kellum, 2008).

\section{Epidemiologia da DRA}

A DRA está associada com alta morbidade e mortalidade e como parte da síndrome de disfunção de múltiplos órgãos (Horkan et al., 2015). Em medicina veterinária, estudos sobre a incidência de casos com DRA ainda são pouco elucidados, devido à variedade de causas e por ser uma enfermidade de progressão e mortalidade rápida e mal diagnosticada.

Alguns estudos pontuais de DRA mostraram que, diferentemente da resolução em humanos, os pacientes caninos e felinos afetados com a DRA apresentam alta mortalidade, girando em torno de $50 \%$ a $60 \%$ (Cobrin et al., 2013). Outro estudo de gatos com DRA demonstrou que a mortalidade foi de 47\% (15/32 gatos) (Worwag e Langston, 2008).

Em uma avaliação retrospectiva realizada na Escola de Veterinária da Universidade da Califórnia (Davis), Estados Unidos, sobre a evolução da DRA em gatos, pode-se concluir que $28 \%$ (37/132) dos gatos avaliados morreram, 30\% (40/132 gatos) foram submetidos à eutanásia e $42 \%$ (55/132 gatos) tiveram que ser submetidos a 30 dias de hemodiálise até receberem alta (Segev et al., 2013).

\section{Classificação da DRA}

Em um consenso na medicina humana, criou-se um sistema de classificação para melhor destacar o conceito de DRA que representa um contínuo dano renal, denominado RIFLE (Risco, Lesão, Falha, Perda e Estádio Final) (Bellomo et al., 2007). Esse sistema tem sido proposto no intuito de avaliar os pacientes e tentar estratificar a extensão e a duração da lesão renal, e consequentemente prever seus resultados clínicos. Os critérios de classificação são baseados ostensivamente em marcadores insensíveis de lesão renal, incluindo mudanças abruptas na taxa de filtração glomerular (TFG), creatinina plasmática e produção de urina (Bellomo et al., 2007; Monaghan et al., 2012a; Iris, 2016). Infelizmente esses critérios de classificação não são tão aplicáveis na rotina clínica de pequenos animais. 
Assim como a Sociedade Internacional de Interesse Renal (IRIS) criou o estadiamento para DRC no intuito de facilitar a consistência de reconhecimento e categorização das previsões de gestão e de resultados para DRC, a IRIS também criou uma classificação para DRA, no intuito de proporcionar um instrumento para 0 reconhecimento precoce, a estratificação terapêutica e avaliação de resultados para DRA em cães e gatos (Iris, 2016)

Ao contrário da classificação segundo a IRIS para DRC, esse esquema não poderia ser aplicado à DRA, por não ser uma enfermidade estável ou que tenha estado de equilíbrio. Pelo contrário, o "grau" representa um momento no decurso da doença e está previsto para mudar à medida que a condição piora, melhora, ou transições de DRC surgem (Iris, 2016).

A Tabela 1 apresenta o esquema de classificação proposto pela IRIS na DRA de cães e gatos, com base na creatinina sérica, formação de urina, e necessidade de terapia de reposição renal (TRR), o qual se destina a facilitar a classificação, a estratificação funcional e a tomada de decisão terapêutica (Iris, 2016).

Tabela 1: Critérios de Classificação da IRIS para DRA.

\begin{tabular}{|c|c|c|}
\hline Grau da DRA & Creatinina Sanguínea & Descrição Clínica \\
\hline Grau I & $\begin{array}{c}<1.6 \mathrm{mg} / \mathrm{dL} \\
(<140 \mu \mathrm{mol} / \mathrm{L})\end{array}$ & $\begin{array}{l}\text { DRA não azotêmico } \\
\text { a. DRA documentada (histórica; clínica; laboratório ou } \\
\text { evidência de imagem da lesão renal aguda; presença de } \\
\text { oligúria/anúria; capacidade de resposta ao volume } * *) \text { e/ou } \\
\text { b. Aumento progressivo de creatinina no sangue de pacientes } \\
\text { não azotêmicos; } \geq 0.3 \mathrm{mg} / \mathrm{dL}(\geq 26.4 \mu \mathrm{mol} / \mathrm{L}) \text { dentro de } 48 \\
\text { horas; oligúria mensurada }(<1 \mathrm{~mL} / \mathrm{kg} / \mathrm{h}) \text { ou anúria durante } 6 \\
\text { horas }\end{array}$ \\
\hline Grau II & $\begin{array}{c}1.7-2.5 \mathrm{mg} / \mathrm{dL} \\
(141-220 \mu \mathrm{mol} / \mathrm{L})\end{array}$ & $\begin{array}{l}\text { DRA suave: } \\
\text { a. DRA documentada e azotemia estática ou progressiva } \\
\text { b. Aumento progressivo da creatinina no sangue de pacientes } \\
\text { azotêmicos; } \geq 0.3 \mathrm{mg} / \mathrm{dL}(\geq 26.4 \mu \mathrm{mol} / \mathrm{L}) \text { dentro de } 48 \text { horas, ou } \\
\text { capacidade de resposta ao volume } * * \\
\text { c. Oligúria mensurada }(<1 \mathrm{~mL} / \mathrm{kg} / \mathrm{h}) \text { ou anúria durante } 6 \text { horas }\end{array}$ \\
\hline Grau III & $\begin{array}{c}2.6-5.0 \mathrm{mg} / \mathrm{dL} \\
(221-439 \mu \mathrm{mol} / \mathrm{L})\end{array}$ & \\
\hline $\begin{array}{l}\text { Grau IV } \\
\text { Grau V }\end{array}$ & $\begin{array}{c}5.1-10 \mathrm{mg} / \mathrm{dL} \\
(440-880 \mu \mathrm{mol} / \mathrm{L}) \\
>10 \mathrm{mg} / \mathrm{dL} \\
(>880 \mu \mathrm{mol} / \mathrm{L})\end{array}$ & $\begin{array}{l}\text { DRA moderada a severa: } \\
\text { a. DRA documentada e severidades crescentes de azotemia e } \\
\text { insuficiência renal funcional }\end{array}$ \\
\hline
\end{tabular}

Os pacientes diagnosticados e classificados com Grau I e II para DRA podem recuperar a função renal adequada dentro dois a cinco dias, prevenir distúrbios azotêmicos e eletrolíticos, o risco de morte é baixo, geralmente, precisando de suporte clínico a curto prazo (Iris, 2016).

Aqueles com graus maiores de DRA cujo grau progride durante a hospitalização, pode exigir terapias de suporte de dias a semanas, de acordo com o início da reparação renal. Os animais com insuficiência renal grave, Grau IV ou V para DRA, podem morrer dentro de cinco a 10 dias, apesar do manejo convencional adequado, ao menos que se faça uso de Terapia Renal Substitutiva (TRS/TRR) por tempo indeterminado (Iris, 2016).

\section{Fisiopatologia da DRA}

A fisiopatologia da DRA é complexa, porém, estudos apontam que existam quatro estádios para descrever essa fisiopatologia, referidos como: iniciação, extensão, manutenção e recuperação. $\mathrm{O}$ insulto ao tecido renal origina a primeira fase de lesão, e a duração desse estádio varia de horas até alguns dias, tempo necessário para que haja o declínio na taxa de filtração glomerular (TFG), sem a presença de sinais clínicos (Monaghan et. al., 2012a).

Independente da causa da injúria inicial, ocorre uma lesão nas células tubulares e indução de isquemia. O quadro hipoxêmico instala-se de forma mais expressiva nas células dos túbulos 
contorcidos proximais e ramo ascendente da Alça de Henle, devido à baixa tensão de oxigênio. Isso leva uma baixa nas concentrações de ATP consequentemente, um aumento do cálcio intracelular, que ativam proteases e fosfolipases. Espécies reativas do oxigênio e radicais livres são então produzidos, deflagrando dano celular. Em resposta, inicia-se uma reação inflamatória renal (Bonventre e Yang, 2011; Bellomo et al., 2012).

A resposta inflamatória tenta proteger o rim da injúria, porém, desencadeia efeitos deletérios como a exposição do citoesqueleto das células tubulares. Com isso, leva a uma perda da função de absorção dos microvilos apicais da borda em escova. Além disso, ocorre uma perda de polaridade das bombas de $\mathrm{Na}+\mathrm{K}+$ e migração das glicoproteínas da posição normal na membrana celular (basolateral) para o citoplasma e membrana apical. Como resultado, o sódio aumenta na mácula densa do túbulo distal (Bonventre e Yang, 2011; Bellomo et al., 2012).

O feedback túbulo-glomerular é ativado e, consequentemente, ocorre uma vasoconstrição arteriolar aferente agravando ainda mais a isquemia renal. A mudança na posição das glicoproteínas nas células tubulares faz com que haja a perda de conexão intercelular e as células se desprendem para o interior luminal tubular (Monaghan et. al., 2012a). A descamação intensa contribui para a obstrução do lúmen, consequentemente o filtrado vaza para o interstício peritubular e aumenta a pressão glomerular, o que diminui a TFG (Bellomo et al., 2012).

O segundo estádio da DRA é o de extensão, no qual há uma amplificação do insulto inicial pela isquemia e resposta inflamatória resultante. Acredita-se que esse estádio tem duração de um a dois dias, visto que as células tubulares sofrem apoptose e necrose, ocasionando uma redução progressiva da TFG. Os mecanismos que culminam com lesão celular continuam, entretanto, a disfunção endotelial com mudança de polaridade e descamação celular parece ter o papel principal nesta fase, resultando em dano morfológico (Monaghan et. al., 2012a).

Em consequência dessa disfunção endotelial, os danos às células do endotélio peritubular provocam maior exacerbação aos agentes vasoconstrictores e reduzem a reatividade aos agentes vasodilatadores, agravando a isquemia e a hipóxia. Os mediadores P-selectina e E-selectina na superfície celular, e a molécula de adesão intercelular 1 (ICAM-1), promovem a interação leucocitária com as células endoteliais renais, aumentando a expressão dos mediadores próinflamatórios e, consequentemente, exacerbam a ativação de mais leucócitos (Bonventre e Yang, 2011; Monaghan et. al., 2012a).

A cascata inflamatória torna-se intensa, com congestão vascular levando ao agravamento da isquemia (Monaghan et. al., 2012a). O dano tubular intensifica-se, nesta fase, sendo ideal a intervenção terapêutica para impedir que o dano celular continue. A duração deste estádio é curta, devido à falta de ferramentas diagnósticas que sejam capazes de detectar os estádios 1 e 2, e, por isso, a injúria progride para o estádio 3 (Bonventre e Yang, 2011).

Na manutenção, a TFG alcança sua taxa mais baixa e se estabiliza. Estima-se que esta etapa dure entre uma e duas semanas. Durante este tempo a apoptose continua, porém o fluxo sanguíneo e o reparo tecidual retornam à normalidade. As complicações urêmicas são mais evidentes nesta fase (Bonventre e Yang, 2011; Monaghan et. al., 2012a).

Por fim, na última fase da DRA, denominada de recuperação, a TFG tem um aumento durante a fase de reparo tecidual maior que durante o estádio 3. Esta pode se reestabelecer ou continuar baixa, resultando em doença renal crônica (DRC). A poliúria pós DRA parece ser um marcador inicial desta etapa de recuperação, podendo durar semanas até meses. Para a completa remissão do dano agudo, é essencial garantir que novas lesões renais não acometam o parênquima renal (Bonventre e Yang, 2011; Monaghan et. al., 2012a).

\section{Etiologia da DRA}

Estudos sobre a causa de DRA em gatos mostrou que em cerca de um terço dos pacientes felinos a causa exata é indeterminada (Monaghan et. al., 2012a; Worwag e Langston, 2008). Porém, existem vários tipos de insultos que são conhecidos por resultar em uma DRA, dentre eles estão fármacos que são utilizados de forma indevida, por exemplo, antiinflamatórios não esteroidais (AINEs), aminoglicosídeos e doxorrubicina (Worwag e Langston, 2008; Segev et al., 2013).

A DRA pode ocorrer também devido a quadros de intoxicação por plantas tóxicas como os lírios e produtos que contenham etilenoglicol em sua composição. Outras possíveis etiologias para IRA incluem: nefropatia isquêmica, nefropatia obstrutiva (obstrução ureteral ou uretral), infecção do trato urinário superior (pielonefrite), neoplasia e 
sepse (Worwag e Langston, 2008; Monaghan et. al., 2012a; Segev et al., 2013).

\section{AINE's}

Os efeitos terapêuticos que as AINEs exercem no organismo são a inibição das enzimas ciclooxigenase, COX-1 e / ou COX-2, para interromper a produção de prostaglandinas. Em um estudo com gatos saudáveis, hemodinamicamente normais e sem comprometimento de lesão renal pré-existente, foi demonstrado que as doses terapêuticas preconizadas para o uso de AINEs não resultaram em quadros de IRA e não afetaram na TFG, demonstrado pela depuração do iohexol em gatos que receberam um ciclo de cinco dias de meloxicam (Goodman et. al., 2009). No entanto, em estados de baixo fluxo sanguíneo renal, as prostaglandinas desempenham um papel crucial na manutenção da função renal e da TFG. As prostaglandinas E2 e I2 exercem efeitos vasodilatadores ao nível do rim e que são importantes na regulação do fluxo sanguíneo, especialmente durante períodos de hipovolemia ou diminuição da perfusão renal (Goodman et al., 2009).

O insulto renal associado ao uso de AINEs é devido a uma interrupção na via da prostaglandina durante os períodos de diminuição do fluxo sanguíneo renal, resultando numa diminuição da perfusão renal e subsequente nefrose isquêmica. Devido a esse mecanismo, todos $\mathrm{S}$ AINEs representam um risco para a função renal, independentemente da seletividade de COX reportada (Monaghan et al., 2012a).

A lesão renal induzida por AINEs é observada com frequência devido à popularidade desses medicamentos e à formulação de comprimidos e líquidos aromatizados desenvolvidos para melhorar a adesão do paciente. Em 2010, a Food and Drug Administration (FDA) dos Estados Unidos da América - EUA anunciou o acréscimo de uma advertência em caixa nos rótulos para o uso de Maxicam ${ }^{\circledR}$ (meloxicam, Boehringer Ingelheim) em gatos, afirmando: "O uso repetido de meloxicam em gatos tem sido associado a insuficiência renal aguda e morte. Não administrar meloxicam injetável ou oral adicional em gatos (Monaghan et. al., 2012a).

O uso de doses excessivas de AINE's, leva ao aparecimento de sinais gastrointestinais em poucas horas após a sua exposição e, além disso, favorece o surgimento de DRA. Esta DRA pode aparecer nas primeiras $24 \mathrm{~h}$, porém alguns estudos mostraram que pode se tornar aparente de 3-5 dias após a exposição. A lesão renal é potencialmente reversível se os medicamentos forem descontinuados e o tratamento for instituído imediatamente (Goodman et. al., 2009).

\section{Aminoglicosídeos}

Muitos medicamentos têm o potencial de induzir a nefrotoxicidade. Existem classes de fármacos dentre os antimicrobianos que já são conhecidos e descritos em literatura por seu efeito nefrotóxico no rim, dentre eles está inserido os aminoglicosídeos. Esse grupo de fármacos pode resultar em uma DRA por lesão direta nas células dos túbulos proximais e conseqüentemente gerar uma necrose tubular aguda (Monaghan et. al., 2012a).

Os fatores de risco para nefrotoxicidade incluem o tipo de aminoglicosídeo, níveis séricos elevados, dose cumulativa, duração e frequência da administração, uso concomitante de furosemida ou outras drogas nefrotóxicas, hipoalbuminemia, hipovolemia e doença renal preexistente (Monaghan et. al., 2012a).

Para reduzir o risco de toxicidade, esses medicamentos devem ser administrados em animais hidratados, com volemia normal sem distúrbios hidroeletrolíticos e ser evitado o uso em pacientes com doença renal preexistente. Deve ser realizada uma monitorização da função renal por meio de urinálise, avaliando o sedimento urinário antes e durante a administração do medicamento (Monaghan et. al., 2012a).

\section{Nefropatia isquêmica}

A isquemia é uma causa comum de lesão renal em pacientes felinos com DRA. Esse tipo de insulto pode resultar devido a eventos hipotensivos (por exemplo anestesia geral), hipovolêmicos (por exemplo, choque, hemorragia, desidratação grave) e doença tromboembólica. Os rins normalmente recebem cerca de 20 a $25 \%$ do débito cardíaco e são altamente suscetíveis a insultos isquêmicos. A autorregulação permite a manutenção da perfusão renal, mesmo quando a pressão arterial média diminui para até $80 \mathrm{mmHg}$ (Abuelo, 2007). Essa manutenção da perfusão ocorre por meio de uma queda na resistência arteriolar-aórtica glomerular, mediada principalmente por prostaglandinas para sustentar a filtração glomerular. A angiotensina II também causa constrição da arteríola eferente, que auxilia na manutenção da filtração glomerular (Abuelo, 2007). 
Se a pressão arterial sistêmica continuar diminuindo, os vasoconstritores endógenos conduzirão a um aumento da resistência arteriolar aferente, com subsequente redução da TFG e do fluxo sanguíneo glomerular pós-perfusão dos túbulos. Eventualmente, o dano tubular resulta em descamação celular e obstrução tubular, e a reabsorção de sódio torna-se prejudicada. Além da lesão causada pela redução do fluxo sanguíneo para os rins, a reperfusão também pode contribuir para a DRA por meio de danos causados pela liberação de oxidantes / radicais livres (Abuelo, 2007).

\section{Nefropatia obstrutiva}

A obstrução do trato urinário pode levar à IRA por meio da oclusão da uretra, ureteres ou pélvis renal. As principais causas de obstrução incluem cálculos, tampões uretrais, coágulos sanguíneos, estenoses ou massas neoplásicas (Reche et. al., 1998). Quando o fluxo de urina é obstruído, ocorre uma rápida redução compensatória da TFG e o desenvolvimento de inflamação e edema. Se esse mecanismo persistir, acaba se desenvolvendo atrofia tubular, fibrose e apoptose. Se a obstrução afeta apenas um rim, e o outro está funcionando normalmente, o rim contralateral se hipertrofia para compensar e o processo pode passar despercebido (Monaghan et. al., 2012a).

\section{Diagnóstico de DRA}

\section{Exame Clínico}

Em relação ao histórico do paciente, tornase necessário uma boa anamnese e um exame clínico completo. Alguns questionamentos devem ser levantados, como tempo de duração da doença, uso de fármacos nefrotóxicos, exposição a toxinas, plantas tóxicas ou componentes alimentares, doenças anteriores e históricos cirúrgicos.

No exame físico os felinos apresentam geralmente apatia, ou letargia e/ou depressão e desidratação. Em casos de curso crônico, os pacientes podem apresentar quadros urêmicos. Essa uremia é manifestada pelo "hálito urêmico" (cheiro semelhante à amônia), ulcerações orais, vômitos e melena.

Os rins encontram-se normais ou aumentados e com algia à palpação. Pacientes com tumor renal ou obstrução ureteral podem ter assimetria no tamanho e forma renal. No entanto, vale ressaltar que pacientes com IRA podem ter DRC preexistente e isso deve ser considerado ao avaliar os achados do exame físico, assim como os resultados laboratoriais e de imagem. Atenção deve ser dada ao tamanho da bexiga urinária para avaliar a obstrução, bem como sugerir a produção de urina.

Em um estudo retrospectivo com 132 gatos diagnosticados com DRA em 2013, foi visto que a maioria dos gatos avaliados apresentava um histórico clínico de letargia, inapetência, anúria e vômitos. Já no exame físico foram percebidas alterações de parâmetros como hipotermia (64\% dos gatos), bradicardia ( $35 \%$ dos gatos), taquipnéia (70\% dos gatos) e renalgia (47\% dos gatos) (Segev et. al., 2013).

Exames hematológicos e bioquímicos

Atualmente, como avaliação laboratorial, utiliza-se a creatinina sérica e a TFG, principais parâmetros laboratoriais utilizados para o diagnóstico da DRA, além da ureia, excreção fracionada de sódio e a proteinúria (Castro et. al., 2016).

Nos gatos com DRA os resultados do hemograma completo geralmente são inespecíficos. Em alguns casos o leucograma pode mostrar uma leucopenia de caráter inflamatório ou de estresse. A anemia não regenerativa é mais típica da DRC, mas pode ser encontrada na DRA, particularmente se houver ulceração gástrica aguda (Monaghan et. al., 2012b). Os resultados do perfil bioquímico podem estar dentro de intervalos dos valores de referência. Porém, estudos mostraram, em relação às enzimas renais (creatinina sérica e uréia), um aumento significativo em série, no grau variado de azotemia (Segev et. al., 2013).

A hipercalemia é comum em pacientes com oligúria ou anúria. No entanto, o potássio também pode estar aumentado quando se tem doenças pósrenais (por exemplo, obstrução urinária ou ruptura do trato urinário); portanto, esse achado não é específico da DRA (Monaghan et. al., 2012b). Alterações em outros valores bioquímicos podem ocorrer, dependendo da etiologia subjacente, como por exemplo a hisperfosfatemia (Segev et. al., 2013).

\section{Urinálise}

A urinálise é um exame laboratorial simples, não invasivo e de baixo custo, sendo uma das mais importantes ferramentas auxiliares ao diagnóstico da DRA. A isoestenúria é a anormalidade mais típica na urinálise de animais com DRA, mas outros achados podem ser úteis na identificação de uma etiologia subjacente, como proteinúria, glicosúria, hematúria, piúria, bacteriúria, 
cristalúria ou cilindros. A cultura e a sensibilidade são recomendadas em todos os pacientes com uma causa desconhecida de IRA antes do tratamento com antibióticos.

A mensuração da concentração das proteínas urinárias avalia a função glomerular e tubular. $\mathrm{O}$ aumento significativo da concentração de proteína na urina serve como diagnóstico na detecção inicial e confirmação da doença renal. Além disso, a proteinúria auxilia na avaliação da eficácia terapêutica e progressão da doença (Price et al., 2005).

Para essa avaliação na rotina clínica, usa-se o método de cálculo da razão proteína/creatinina urinárias $(\mathrm{RPCu})$. Este parâmetro somente pode ser considerado quando o sedimento urinário está inativo. Em pacientes hígidos, até $150 \mathrm{mg}$ de proteína por dia é considerado um achado normal. De acordo com a IRIS, considera-se que em gatos, a razão de proteína na urina seja menor que 0.2 (não-proteinúricos), com valores limites entre 0,2 a 0,4 , e valores acima destes citados confirmam a proteinúria (Iris, 2016).

A avaliação da excreção fracionada eletrólitos é um exame laboratorial de aplicação bastante útil na rotina clínica de pacientes com DRA. Este exame, tem a capacidade de avaliar a função tubular renal, por meio do cálculo entre o percentual que é excretado em relação à concentração eletrolítica sérica do mesmo, e é feita a correção baseada na taxa de filtração e na excreção constante de creatinina (Castro et. al., 2016).

\section{Exames de Imagem}

A radiografia abdominal e a ultrassonografia podem ser úteis para caracterizar a etiologia da lesão. A radiografia é útil para avaliação do tamanho e forma do rim, e pode ser usada para identificar cálculos radiopacos no trato urinário. A ultrassonografia abdominal pode ser usada para avaliar mais a obstrução (particularmente com cálculos não radiopacos), neoplasia ou sinais de pielonefrite. A dilatação pélvica renal moderada pode ser detectada em cães e gatos com função renal clinicamente normal, mas o tamanho pélvico aumentará com insuficiência renal, pielonefrite ou obstrução ao fluxo de saída (Monaghan et. al., 2012b; Lamb et. al., 2018).

Pielografia anterógrada ou tomografia computadorizada pode delinear ainda mais a presença de uma obstrução ureteral, se não visível na ultrassonografia, como é o caso de cálculos sanguíneos solidificados secos ou de coágulos sanguíneos (Lamb et. al., 2018).

\section{Aferição da Pressão Arterial}

A aferição da pressão arterial e o exame de fundo de olho são exames importantes para complementar o diagnóstico de problemas renais, e devem ser realizados na rotina clínica de pequenos animais. Dependendo da causa subjacente e da gravidade da doença, os pacientes com DRA também podem sofrer quadros de hipotensão, o que pode exacerbar a lesão renal (Monaghan et. al., 2012b; Cole et. al., 2017).

\section{Biomarcadores}

Os biomarcadores ou marcadores biológicos consistem em medidas laboratoriais capazes de refletir determinada função normal, alterações fisiológicas ou uma resposta a um agente farmacológico. Mais de 20 biomarcadores de IRA já foram estudados e são extremamente valiosos, especialmente na lesão isquêmica comum na sepse e na desfunção cardiopulmonar (Castro, 2016).

Um dos biomarcadores bastante estudado é a Gama glutamiltransferase urinária (GGTu). A GGT é uma enzima urinária que se localiza na borda em escova dos túbulos contorcidos proximais e na alça de Henle dos néfrons, sendo uma molécula muito grande para ser filtrada, fisiologicamente, pelos glomérulos. Quando a GGTu está presente na urina indica uma possível injúria, usualmente associada a lesão ou necrose epitelial tubular. Normalmente, aumentos de duas a três vezes o valor basal indicam lesão do epitélio tubular, sendo, por isso, considerada um marcador precoce de dano tubular renal (Castro et. al., 2016).

Outro biomarcador que está sendo bastante estudado em gatos com nefropatia obstrutiva é o Nacetil-beta-d-glicosaminidase (NAG) urinário, é uma enzima presente nos túbulos proximais renais, utilizada na medicina humana e veterinária como biomarcador para detecção de danos tubulares renais (Panboon et al., 2017).

Por fim, um desses biomarcadores que já está bastante elucidado e utilizado na rotina clínica veterinária é a dimetilarginina simétrica (SDMA). O SDMA é uma forma metilada do aminoácido arginina, que é liberada para a circulação durante a degradação da proteína e é excretada pelos rins (Hall et al., 2014a). Um estudo demonstrou que o SDMA aumenta, em média, quando há uma perda de $40 \%$ da função renal, e consistentemente, meses anteriores à creatinina em gatos com ocorrência 
natural da DRC (Hall et al., 2014a). O SDMA não é influenciado pela massa corporal magra e, portanto, reflete com mais precisão a TFG em gatos geriátricos, ao contrário da creatinina sérica, que diminuiu com envelhecimento (Hall et al., 2014b).

O estadiamento da DRC pela IRIS, um aumento do SDMA acima de $14 \mathrm{mg} / \mathrm{dL}$ sugere uma redução da função renal e pode ser uma razão para considerar a DRC em um gato com valores de creatinina $<1,6 \mathrm{mg} / \mathrm{dL}$ (Iris, 2016).

\section{Tratamento da DRA}

O tratamento da DRA deve ser iniciado o mais cedo possível e requer monitoramento intensivo do paciente. Nos casos em que se conheça a causa da DRA, estas devem ser eliminadas e o tratamento deve ser realizado direcionado aos sinais consequentes à uremia aguda. Fármacos nefrotóxicos devem ser descartados (Monaghan et al, 2012b).

A fluidoterapia intravenosa é essencial no tratamento da DRA, porém deve ser realizada e monitorada adequadamente. Inicialmente, é comum o uso de uma solução substituta (como PlasmaLita A, solução de Ringer com Lactato ou Normosol-R) (Monaghan et al, 2012b). Gatos que apresentem hipercalemia grave pode-se considerar o uso de solução salina normal a $0,9 \%$. Como a maioria dos gatos com a IRA também apresenta algum grau de desidratação, é importante realizar a hidratação para garantir fluxo sanguíneo renal adequado. Os fluidos intravenosos devem ser administrados para substituir as perdas em curso e potencialmente estimularem a diurese (Monaghan et al, 2012b).

Há controvérsia em relação à diurese forçada em pacientes com IRA visto que há chance de elevar a morbidade associada à sobrecarga de fluído. Assim, é importante realizar a fluidoterapia com volume adequado ao paciente em questão, evitando a hiperidratação. A sobrecarga hídrica está associada ao aumento da morbidade e mortalidade em pacientes com DRA humana e, provavelmente, tem impactos semelhantes em pacientes veterinários. A duração da fluidoterapia varia conforme a resposta do paciente e considerase restabelecido o animal hidratado que apresente, por meio de exames laboratoriais, valores renais normais ao longo de 24 horas (Monaghan et al, 2012b).
Distúrbios eletrolíticos e ácido-base

Distúrbios eletrolíticos são comuns em pacientes com DRA, sendo os mais frequentemente relatados a hiperfosfatemia e a hipercalemia (Bellomo et al., 2012). O tratamento da hiperfosfatemia é realizado por meio da fluidoterapia e, em pacientes que estejam comendo, administração de ligantes de fosfato enteral, que diminuem a absorção do mineral presente na dieta (Monaghan et al, 2012b).

A hipercalemia pode ser revertida com a administração endovenosa lenta de gluconato de cálcio $(0.5-1 \mathrm{ml} / \mathrm{kg})$, que antagoniza os efeitos do potássio na membrana das fibras cardíacas, controlando a condutância cardíaca. Para tanto, deve ocorrer o monitoramento cardíaco através de eletrocardiograma. Outras formas para reduzir a hipercalemia são administrar insulina, dextrose (0.25-0.5 g/kg IV), bicarbonato de sódio (1-2 mEq $/ \mathrm{kg}$ IV durante 10-20 minutos), terbutalina (0.01 $\mathrm{mg} / \mathrm{kg}$ subcutânea ou intramuscular), insulina e terbutalina. O objetivo é reduzir os níveis séricos de potássio e promover cardioproteção (Monaghan et al, 2012b).

Geralmente, após o reestabelecimento da diurese do paciente devido à hidratação, o distúrbio ácido-base é, aos poucos, corrigido. Se mesmo com a hidratação após fluidoterapia o $\mathrm{pH}$ do paciente for menor que 7,1 ou TCO2 for $<12 \mathrm{mEq} / 1$, o tratamento com bicarbonato de sódio pode ser considerado (Monaghan et al, 2012b).

\section{Oligúria e anúria}

Caso a diurese não seja restabelecida após a hidratação, podem ser administrados diuréticos furosemida $(1-2 \mathrm{mg} / \mathrm{kg}$ IV bolus ou $0,25-1$ $\mathrm{mg} / \mathrm{kg} / \mathrm{h} \mathrm{IV}$ por infusão contínua), manitol (0,5-1 $\mathrm{g} / \mathrm{kg}$ IV bolus ou $1-2 \mathrm{mg} / \mathrm{kg} / \mathrm{min}$ IV por infusão contínua) ou agonistas dopaminérgicos fenoldopam $(0,1-1 \mu \mathrm{g} / \mathrm{kg} / \mathrm{min}$ IV por infusão contínua) -para promover o fluxo urinário adequado. Gatos que não consigam responder aos tratamentos em até 12 horas, devem ser submetidos à TRR (diálise) (Monaghan et al, 2012b).

\section{Hipertensão}

A hipertensão é uma das consequências frequentes da DRA (Langston, 2002). Assim, é importante aferir a pressão arterial sistêmica desses pacientes. $\mathrm{O}$ tratamento de pacientes hipertensivos 
pode ser realizado com amlodipina, um bloqueador de canais de cálcio $(0,625$ a $1,25 \mathrm{mg} / \mathrm{dia}$, SID por via oral) (Cole et al., 2017). O uso de inibidores da ECA, como benazepril ou enalepril, só é recomendado para pacientes que já se recuperaram da DRA (Monaghan et al, 2012b).

\section{Sequelas gastrointestinais}

Devido à uremia alguns gatos podem apresentar sequelas gastrointestinais como vômito, anorexia e úlceras gástricas, devendo serem tratadas. Podem ser administrados antieméticos metoclopramida ( $1 \mathrm{mg} / \mathrm{kg} \mathrm{VO})$, dolasetrona $(0,6$ $\mathrm{mg} / \mathrm{kg}$, IV, SC ou VO), ondansetrona $(0,1-0,2$ $\mathrm{mg} / 1 \mathrm{~kg} \mathrm{VO}, \mathrm{IV})$, e inibidores de secreção gástrica - famotidina $(0,1-0,4 \mathrm{mg} / \mathrm{kg} \mathrm{VO}, \mathrm{SC}, \mathrm{IM})$, ranitidina $(2,5 \mathrm{mg} / \mathrm{kg} \quad \mathrm{IV}, \quad 3,5 \mathrm{mg} / \mathrm{kg} \quad \mathrm{VO})$, omeprazol $(0,7 \mathrm{mg} / \mathrm{kg} \mathrm{VO})$. Em pacientes com anorexia, a alimentação via sonda deve ser considerada, principalmente via enteral, sendo a dose diária nutricional recomendada (Monaghan et al, 2012b).

\section{Terapia de Reposição Renal (TRR)}

A TRR é indicada para pacientes que não apresentem resposta às intervenções médicas previamente realizadas, como oligúricos ou anúricos, com hipercalemia refratária, entre outras. O objetivo é estabilizar o quadro do paciente até a recuperação da função renal ou, ainda, eliminar substâncias tóxicas do organismo (Langston, 2002). Infelizmente, o acesso a esse tipo de terapia ainda é limitado.

Conforme relatado por Monaghan et al (2012b), existem três tipos de TRR: diálise peritoneal (DP), hemodiálise intermitente (HDI) e terapia de reposição renal contínua (TRRC). A DP é realizada através da colocação de um cateter peritoneal ligado a um sistema fechado de coleta, permitindo a infusão de dialisato, que permanece por um período de tempo no espaço peritoneal e depois é removido.

HDI é feita através de um filtro extracorporal que remove os líquidos e solutos excedentes, corrigindo o equilíbrio ácido-base e valores de eletrólitos. A sessão dura entre 4 e 8 horas e é indicada para rápida remoção de toxinas, nos casos de DRA refratária ou para terapia de longo prazo (Monaghan et al, 2012b). O que difere a TRRC da HDI é o tempo de filtração, que é mais lento, assemelhando-se à função renal. A TRRC é realizada 24 horas por dia, sendo ideal para pacientes hemodinamicamente instáveis, especialmente no início do manejo terapêutico (Langston, 2010; Monaghan et al, 2012b).

\section{Considerações Finais}

A DRA é uma enfermidade emergencial grave e bastante frequente na clínica de felinos, possuindo etiologia multifatorial e muitas vezes difícil identificação da causa com diagnóstico geralmente em estádios avançados da lesão renal tornando o prognóstico reservado. $\mathrm{O}$ manejo adequado do paciente requer monitoramento intenso e terapia específica para o caso clínico. Apesar dos procedimentos adequados, muitos pacientes com elevado grau de lesão renal não respondem ao tratamento. Com isso, são necessários mais estudos que venham a contribuir com o diagnóstico precoce da DRCA, viabilizando o tratamento precoce desta doença assim evitando sua progressão e minimizando o dano renal do paciente.

\section{Referências}

Abuelo, J.G. Normotensive ischemic acute renal failure. The New England Journal of Medicine, 357(8): 797-805, 2007.

Bellomo, R.; Kellum, J.A.; Ronco, C. Acute kidney injury. The Lancet, 380(9843): 756-766, 2012.

Bonventre, J.V.; Yang, L. Cellular pathophysiology of ischemic acute kidney injury. The Journal of Clinical Investigation, 121(11): 4210-4221, 2011.

Castro, L.T.S.; Dall'Agnol, M.; Araujo, M.S.; Fioravanti, M.C.S.; Ariza, P.C. Biomarcadores no diagnóstico precoce da injúria renal aguda. Enciclopédia Biosfera, 13(23): 216-241, 2016.

Cobrin, A.R.; Blois, S.L.; Kruth, S.A.; AbramsOGG, A.C.; Dewey, C. Biomarkers in the assessment of acute and chronic kidney diseases in the dog and cat. The Journal of Small Animal Practice, 54(12): 647-655, 2013.

Cole, J.; Jepson, R.; Humm, K. Systemic hypertension in cats with acute kidney injury. Journal of Small Animal Practice, 58(10): 577-581, 2017.

Goodman, L.A.; Brown, S.A.; Torres, B.T.; Reynolds, L.R.; Budsberg, S.C. Effects of meloxicam on plasma iohexol clearance as a marker of glomerular filtration rate in conscious healthy cats. American Journal of Veterinary Research, 70: 826-830, 2009.

Hall, J.A.; Yerramilli, M.; Obare, E.; Yerramilli, M.; Jewell D.E. Comparison of sérum concentrations of symmetric dimethylarginine 
and creatinine as kidney function biomarkers in cats with chronic kidney disease. Journal of Veterinary Internal Medicine, 28(6): 16761683, 2014a.

Hall, J.A.; Yerramilli, M.; Obare, E.; Yerramilli, M.; Yu, S.; Jewell, D.E. Comparison of serum concentrations of symmetric dimethylarginine and creatinine as kidney function biomarkers in healthy geriatric cats fed reduced protein foods enriched with fish oil, L-carnitine, and mediumchain triglycerides. Veterinary Journal, 202(3): 588-596, 2014b.

Horkan, C.M.; Purtle, S.W.; Mendu, M.L.; Moromizato, T.; Gibbons, F.K.; Christopher, K.B. The association of acute kidney injury in the critically ill and postdischarge outcomes: a cohort study. Critical Care Medicine, 43(2): 354-64, 2015.

Kellum, J.A. Acute Kidney Injury. Critical Care Medicine, 36(4 suppl): S141-145, 2008.

Lamb, C.R.; Cortellini, S.; Halfacree, Z. Ultrasonography in the diagnosis and management of cats with ureteral obstruction. Journal of Feline Medicine and Surgery, 20(1): 15-22, 2018.

Langston, C. Hemodialysis in dogs and cats. Compendium on Continuing Education for the Practicing Veterinarian, 24(7): 540-549, 2002.

IRIS. International Renal Interest Society. IRIS Grading of Acute Kidney Injury (AKI), de 2016. Disponível em: <http://www.iriskidney.com/guidelines/grading.html>. Acesso em: 21 jan. 2018.

Monaghan, K.; Nolan, B.; Labato, M. Feline acute kidney injury -1 . Pathofisiology, etiology and etiology-specific management considerations. Journal of Feline Medicine and Surgery, 14(11): 775-784, 2012a.
Monaghan, K.; Nolan, B.; Labato, M. Feline acute kidney injury -2 . Approach to diagnosis, treatment and prognosis. Journal of Feline Medicine and Surgery, 14(11): 785-793, 2012b.

Nagai, J.; Tanaka, H.; Nakanishi, N.; Murakami, T.; Takano, M. Role of megalin in renal handling of aminoglycosides. American Journal of Physiology. Renal Physiology, 281(2): F337-344, 2001.

Panboon, I.; Asawakarn, S.; Pusoonthornthum, R. Urine protein, urine protein to creatinine ratio and $\mathrm{N}$-acetyl-beta-D-glucosaminidase index in cats with idiopathic cystitis vs healthy control cats. Journal of Feline Medicine and Surgery, 19(8): 869-875, 2017.

Price, C.P.; Newall, R.G.; Boyd, J.C. Use of protein: creatinine ratio measurements on random urine samples for prediction of significant proteinuria: a systematic review. Clinical Chemestry, 51(9): 1577-1586, 2005.

Reche Jr., A.; Hagiwara, M.K.; Mamizuka, E. Estudo clínico da doença do trato urinário inferior em gatos domésticos de São Paulo. Brazilian Journal of Veterinary Research and Animal Science, 35(2): 69-74,1998.

Segev, G.; Nivy, R.; Kass, P.H.; Cowgill, L.D. A Retrospective Study of Acute Kidney Injury in Cats and Development of a Novel Clinical Scoring System for Predicting Outcome for Cats Managed by Hemodialysis. Journal of Veterinary International Medice, 27: 830839, 2013.

Worwag, S.; Langston, C.E. Acute intrinsic renal failure in cats: 32 cases (1997-2004). Journal of the American Veterinary Medical Association, 232(5): 728-732, 2008. 